\title{
Hydrocephalus and Dandy- Walker Malformation:
}

\section{a review}

\author{
Patricia Alessandra Dastoli', Jardel Mendonça Nicácio', Marcos Devanir Silva da Costa ${ }^{1}$, \\ Italo Capraro Suriano', Marcia Cristina da Silva², Sergio Cavalheiro'
}

Received: 10 May 2020 / Published: 07 October 2020

\begin{abstract}
Introduction: Dandy Walker malformation (DWM) is characterized by agenesis or hypoplasia of the cerebellar vermis, cystic dilatation of the fourth ventricle leading to an increase of the posterior fossa and superior dislocation of the lateral sinus, tentorium and torcula. Although it is the commonest posterior fossa malformation, its pathogenesis is still not fully understood, making the differential diagnosis with other posterior fossa malformations difficult and as a result the choice of therapeutic strategy.

Material and methods: An extensive review of the literature relating to Dandy Walker malformation was performed. Historical, genetic, embryologic, epidemiologic, clinical and radiological presentation, treatment and prognosis were revised.

Conclusion: The correct diagnosis of Dandy Walker malformation can be made through careful interpretation of magnetic resonance imaging (MRI) and cerebrospinal fluid (CSF) flow studies. The choice of hydrocephalus treatment depends on whether there is aqueduct stenosis. And, although ventriculoperitoneal (VP) shunts have been the treatment of choice for many years, neuroendoscopic techniques such as endoscopic thirdventriculostomy and stent placement are being frequently performed lately.
\end{abstract}

Key words: Dandy-Walker malformation, Hydrocephalus, CNS malformation, Shunts, Neuroendoscopy

\footnotetext{
${ }^{1}$ Department of Neurology and Neurosurgery, Universidade Federal de São Paulo, São Paulo- SP, Brazil

${ }^{2}$ Department of Neurosurgery, João XXIII Hospital, Belo Horizonte, MG, Brasil

To whom correspondence should be addressed: Patricia Alessandra Dastoli , MD, PhD [E-mail: paty.dastoli@uol.com.br]
}

Journal homepage: www.sbnped.com.br

\section{Introduction}

From the first description by Sutton in 1887 to the present time, much is still discussed about DandyWalker malformation (DWM)[1]. Difficulties persist in differentiating DWM from other cystic malformation of the posterior fossa. At times, Blake's pouch cyst, Dandy-Walker variants, mega cisterna magna or even arachnoid cysts of the posterior fossa are mistakenly diagnosed as DWM [2].

Consequently, the diagnostic error determines the incorrect choice of treatment of the hydrocephalus associated to this malformation.

The goal of this review of the literature is to promote up-to-date understanding of DWM, its definition, the cerebrospinal (CSF) circulation changes that result in hydrocephalus and differential diagnosis in imaging studies. Lastly, in light of these concepts, the different strategies for the treatment of hydrocephalus employed in the last decades are discussed.

\section{Material and Methods}

An extensive review of the literature relating to Dandy Walker malformation was performed with no date limit. MEDLINE database was accessed using PubMed Central at the U.S. National Institutes of Health's National Library of Medicine (NIH/NLM),(http://www.ncbi.nlm.nih.gov/pubmed/) The search was done with the following keywords and their associations : Dandy- Walker malformation, Dandy-Walker complex, Dandy-Walker syndrome, Dandy- Walker variant, posterior fossa cysts, posterior fossa malformations, posterior fossa arachnoid cyst, mega cisterna magna, Blake's pouch cyst, epidemiology, cerebellar malformations, cerebellar vermis, embryology, foramen of Luschka, foramen of 
Magendie, aqueduct, hydrocephalus, diagnoses, neuroradiology, surgical treatment, shunts, ventriculoperitoneal shunt, cystoperitoneal shunt, endoscopic third ventriculostomy, aqueductal stent, aqueductoplasty and outcome. A total of 55 articles were available for analysis (English and French).

\section{Results}

\section{History}

Sutton made the first description of the association between hydrocephalus, a large posterior fossa cyst and hypoplasia of the cerebellar vermis in 1887 [1]. Dandy and Blackfan in 1914 reported the case of a 13 month-old girl with severe hydrocephalus associated with a dilated fourth ventricle, hypoplasia of the cerebellar vermis and absence of the foramina of Luschka and Magendie (Fig. 1). They believed that the closure of these foramina impeded the CSF flow from the fourth ventricle to the subarachnoid space, causing hydrocephalus. The theory of atresia was then established [3].

Taggart and Walker reported in 1942 the case of three children presenting with hydrocephalus associated to atresia of the foramina of Luschka and Magendie[4]. Nonetheless, in 1941 Sahs et al. already called attention to the case of a patient presenting with congenital anomaly of the cerebellar vermis, patent Luschka foramina without hydrocephalus, features are reminiscent of the alterations described by Dandy in $1914[4,5]$.

It was Benda in 1954 who, after examining six patients presenting with hydrocephalus and a cystic dilation of the posterior fossa, indicated that this dilation was not always associated with stenosis of the foramina of Luschka and Magendie. Benda suggested that DWM was the result of a failure of the embryonic regression of the posterior medullary velum, associated with a congenital absence of the cerebellar vermis, resulting in the formation of a cyst at the caudal portion of the fourth ventricle that could explain the hydrocephalus. $\mathrm{He}$ proposed that the hydrocephalus associated with the cerebellar vermis hypoplasia and cystic dilation of the fourth ventricle be named Dandy-Walker malformation. He was the first to emphasize that the atresia of the cerebellar foramina was not an essential characteristic of the malformation [6].

In 1977 Gardner at al. proposed the hydrodynamic theory that states that a hypertrophy of the posterior fossa choroid plexus and ensuing increase of CSF production would lead to pouching of the anterior membranous area, cystic enlargement of the fourth ventricle and elevation of the tentorium [7].

Studies from Gibson, D'Agostino and Hart defined the characteristic triad of DWM as we know it today constituted by:

a) complete or partial agenesis of the cerebellar vermis; b) cystic dilation of the fourth ventricle;

c) enlargement of the posterior fossa with superior displacement of the transverse sinus, torcula and tentorium $[2,8,9,10]$.

This triad is usually associated with hydrocephalus, though hydrocephalus should be considered more of a complication instead of a fundamental element of the malformation, as pointed out by Raybaud in 1982 [11].

In 1962 Gosset et al. first utilized the term mega cisterna magna to describe the findings from a series of adult patients showing very enlarged cisterns that they interpreted as the result of cerebellar atrophy. Nowadays the term is widely used to describe a large retrocerebellar space with normal vermis and cerebellar hemispheres [12].

Harwood-Nash and Fish introduced in 1976 the term "Dandy-Walker variant" to describe a condition with posterior evagination of the anterior membranous area (AMA), partial agenesis of the vermis and normal sized posterior fossa. In 1982 Raybaud used the same term to describe a malformation with varying degrees of cerebellar vermis agenesis and an increased fourth ventricle that communicated with the subarachnoid space. Raybaud then considered a Dandy-Walker malformation only cases in which there was no evidence of communication between the fourth ventricle and the subarachnoid space $[11,13]$.

Finally, in 1989 Barkovich et al. tried to stablish a clear distinction between DWM, Dandy-Walker variant and mega cisterna magna, according to magnetic resonance imaging analysis. They considered the altered structures represented a continuum of developmental anomalies of the posterior fossa and grouped them under the term "Dandy-Walker complex". This classification is still widely utilized in the clinical setting and, unfortunately, leads to much diagnostic confusion $[2,14]$. Barkovich's classification divides the cystic malformations of the posterior fossa in two basic categories:

1. Dandy-Walker Complex: in this group the posterior fossa collections clearly communicate with the fourth ventricle. Depending on whether the cerebellar vermis is present, they are classified as type A or B:

- type A: hypoplasia and/or rotation of the cerebellar vermis does not allow it to be visible. This group includes the classic malformation of Dandy and Blackfan and those malformations defined as DandyWalker variant by Harwood-Nash and Raybaud.

- type B there is an interposition between the cerebellar vermis and an enlarged cisterna magna. There is ample retrocerebellar space that communicates with the fourth ventricle and the posterior fossa is of normal size. It corresponds to those anomalies defined as mega cisterna magna $[2,14]$.

According to these criteria, the Blake's pouch cyst is also included in this subgroup, a cystic malformation 
of the posterior fossa that would be described by Tortori and Donati in 1996 [2,14,15].

2. Posterior fossa arachnoid cysts: this group includes the retrocerebellar collections that do not communicate directly with the fourth ventricle. They may compress the cerebellum and obstruct the aqueduct, leading to hydrocephalus. The clinical presentation of these cysts is very different and the cognitive prognosis is much better that of patients with DWM $[2,14,15]$.

In 1993 Kolias et al. adopted the term "cerebellar vermian hypoplasia" to describe a group of malformations characterized by normal size posterior fossa, varying degrees of vermis and cerebellar hypoplasia, a retrocerebellar space that communicated freely with a fourth ventricle of normal size or moderately dilated and no hydrocephalus [16].

Concurrently to the anatomic descriptions of these malformations, descriptions of another posterior fossa collection appeared in the literature - the Blake's pouch cyst or persistence of the Blake's pouch or Blake's cyst. It was initially described as a retrocerebellar cyst by Gilles in 1971 or retrocerebellar arachnoid pouch by Raybaud in 1982[11]. Only in 1996 did Tortori and Donati classified it as an entity independent from the Dandy-Walker complex and proposed a classification based on the embryogenesis of the cerebellum and fourth ventricle $[11,15,17]$. According to these authors, developmental anomalies of the anterior membranous area (AMA) give origin to DWM or Dandy-Walker variant, while developmental anomalies of the posterior membranous areas (PMA) result in mega cisterna Magna or persistence of Blake's pouch [15].

Blake's pouch is a transient cystic formation of the posterior membranous area that occurs during the embryonic period and initially does not communicate with the surrounding subarachnoid space [15]. It was described by Blake in 1900, who noticed an evagination in the midline of the roof of the fourth ventricle of a human embryo, limited by ependyma. This evagination extended posterior and superiorly inside the inferior primitive meninges to the cerebellum. In its walls there were histological elements of glia, ependyma and choroid plexus [18]. The posterior membranous area (PMA) gives rises to the tela choroidea of the fourth ventricle before the opening of the foramen of Magendie. If there is a delay or non-opening of this foramen, the cystic formation does not regress. The persistence of this cystic formation is called Blake's pouch cyst. Since this cyst does not communicate with the cistern, there is tetraventricular hydrocephalus that is one of the diagnostic criteria of this malformation [19]. As there is no communication between the fourth ventricle and the subarachnoid space of the midline, many authors do not classify it as part of the Dandy-Walker complex. Yet other authors state that Blake's pouch cyst and mega cisterna magna represent less severe anomalies inside the DWM continuum. For these reasons, until today there is great difficulty in stablishing the differential diagnosis of these cystic lesions of the posterior fossa, with confusing diagnosis and inappropriate use of terminology, culminating with the wrong choice of therapeutic strategy[2,19].

\section{Genetics}

DWM can be associated with several genetic syndromes and abnormalities. In the last decades, several genetic loci were described in the pathogenesis of DWM and other related posterior fossa malformations, such as Dandy-Walker variant and mega cisterna magna. The first locus connected to DWM was identified by Grinberg et al in 2004. In individuals with deletion of $3 q 2$, the cause of DWM may be the loss of heterozygotes genes $\mathrm{Z1C} 1$ and $\mathrm{Z} 1 \mathrm{C} 4$. It was demonstrated that $\mathrm{Z} 1 \mathrm{C} 1$ is related to cerebellar development in mice; and severely affected mice in both Z1C1 and Z1C4 loci that had a markedly smaller cerebellar vermis did not survive for a long after birth[20].

The gene Fokhead box (FOXC1), situated in the chromosome 6p25,3, is also important in the normal cerebellar development and is associated with DWM and other posterior fossa malformation. This gene is expressed in the adjacent posterior fossa mesenchyme early in the CNS formation. It is necessary for directing migration and differentiation of the roof plate and rhombic lip derivatives. Its loss, and consequently the defects of mesenchymal signaling, can result in cerebellar malformations compatible with DWM, Dandy-Walker variant and mega cisterna magna [20].

Similar posterior fossa malformations have also been described in patients presenting tetrasomy $9 p$, with deletion of $13 q$ and deletion of $2 q 36.1$. Deletions or duplications of chromosome $7 \mathrm{p} 21.3$ also relate to cerebellar malformations, associated with anomalous expression of genes NDUFA4 and PHF14 [20].

\section{Embriology of the Cerebellum and Fourth ventricle}

The understanding of the main stages of embryonic development of the cerebellum and fourth ventricle is fundamental for the understanding of the posterior fossa malformations [21].

In the descriptions of DWM from the beginning of the 20th century, failure of the opening of the foramina of Luschka and Magendie was the explanation for the genesis of the malformation and the hypoplasia of the cerebellar vermis would be a consequence of chronic compression from the cyst $[3,4,22]$.

It is known now that DWM is the result of dysgenetic development of the roof of the rhombencephalon at the level of the anterior membranous area. The role of foraminal obstruction in the genesis of DWM is much more complex[20]. 
During the fourth gestational week, after the fusion of the neural tube and closure of the rostral neuropore, the 3 primary cerebral vesicles arise: forebrain (prosencephalon), midbrain (mesencephalon) and hindbrain (rhombencephalon). The embrionary brain grows rapidly and the neural tube acquires a zig-zag shape $[23,24]$. Two flexures develop: a cranial and a cervical. The rhombencephalon corresponds to the region between the cephalic and cervical flexure. Around the sixth week of gestation, the pontine flexure appears and divides the rhombencephalon into 2 secondary vesicles: the superior vesicle called metencephalon and the inferior vesicle, called myelencephalon [21]. The pons develops from a thickening of the floor and lateral walls of the metencephalon. In addition, the brainstem is formed from the floor and lateral walls of the myelencephalon. The cerebellum originates predominantly from the hindbrain, though it receives a small contribution from the alar plates of the distal third of the mesencephalon, that originates the vermis. The remainder, the cerebellar hemispheres, originate from the alar plates of the metencephalon (cranial portion of the rhombencephalon). The cavity of the hindbrain will correspond to the future fourth ventricle $[21,24,25]$.

After the formation of the pontine flexure, the cranial and caudal extremities of the fourth ventricle approximate dorsally and the roof of the fourth ventricle is pushed inside the cavity, whereas the alar laminae are splayed laterally because of the bending of the pons and eventually lie dorsolateral to the basal laminae. In this way, the roof plate corresponding to the roof of the developing fourth ventricle remains thin and expands transverse and dorsally. The margins of the fourth ventricle angle obliquely towards the midline in its superior and inferior portion, starting from the central region, where the margins remain amply separated. The surrounding mesenchymal tissue insinuates into the fold at the level of the floor of the fourth ventricle and forms the plica coroidalis, that is the precursor of the choroid plexus $[21,25,26]$.

The roof of the fourth ventricle is divided by the plica coroidalis in 2 areas: the anterior membranous area (AMA) superiorly and the posterior membranous area (PMA) inferiorly. The lateral margins of the AMA thicken due to intense neuroblastic proliferation activity that gives origin to 2 lateral plates known as the rhombic lips. This process initiates approximately between the fourth and the sixth gestational week [21].

The rhombic lips (the developing cerebellum) increase in size and get closer to each other, fusing at the midline. This growth in the rostral half of the fourth ventricle makes the rhombic lips overlap the pons and the medulla. As the rhombic lips develop, the AMA regresses and is completely incorporated into the developing choroid plexus (Fig. 2) [21]. The growth and backward extension of the cerebellum pushes the choroid plexus inferiorly and the PMA also regresses. Subsequently, a marked caudal protrusion of the fourth ventricle develops, leading to an expansion of the PMA like the finger of a glove. This transient protrusion is known as Blake's pouch. The Blake's pouch consists of ventricular ependyma surrounded by condensation of the mesenchymal tissues. The Blake's pouch is initially a closed cavity that does not communicate with the surrounding subarachnoid space of the cisterna magna $[21,22,24,25]$.

The network between the vermis and the Blake's pouch progressively becomes condensed, whereas the other portions about the evagination become rarified. Thus a permeabilization of the Blake's pouch occurs, which then forms the foramen of Magendie. The formation of the cisterna magna presumably occurs at approximately 6 weeks of gestation, which is in communication with the fourth ventricle via foramen of Magendie. The precise time of the opening of the foramen of Magendie is not established. However, persistence of the Blake's pouch has been demonstrated into the 4th gestational month. The foramina of Luschka also probably appear late into the 4th month of gestation $[11,21,25,26,27,28,29]$.

Failure of incorporation of the AMA into the choroid plexus determines the persistence of this membrane between the caudal edge of the developing vermis and the cranial edge of the developing choroid plexus. The pulsations CSF causes the AMA to balloon out into a cyst that displaces the hypoplasic vermis superiorly anticlockwise. PMA can persist unopened or become patent, accounting for the reportedly variable patency of the foramen of Magendie and association of hydrocephalus. Global enlargement of the PF may result from arrested development of the tentorium, straight sinus and torcula, with failure of migration of the straight sinus from the vertex to the lambda, possibly because of the abnormal distention of the 4th ventricle $[21,29]$.

\section{Epidemiology and Clinical Features}

The estimated prevalence of DWM and variants is around 1:25000 to 1:30000 live births. In the United States, the prevalence is 1:35000 live births. According to ORPHANET, the European portal of rare diseases registry, the prevalence of DW in Europe is 1:100000 live births. Theses malformations account for approximately 1 to $4 \%$ of cases of hydrocephalus and familial cases are rare [30].

Today, the advancement of diagnostic techniques has made early diagnosis of DWM possible and the proportion of cases diagnosed prenatally has increased. In the EUROCAT (European Surveillance of Congenital Anomalies) study population from 2019, the general prevalence of DWM is $6.79 / 100.000$ and Dandy-Walker variant of $8.85 / 100.000$. This study registers congenital anomalies in Europe. On the other hand, the prevalence among livebirths is $2.74 / 100.000$. One should note that the prenatal detection index of DWM in Europe is around $90 \%$. Prenatal diagnosis and the awareness of the prognosis of these patients can be of help in the 
postnatal treatment, as well as influence the parents' decision of whether to continue with the pregnancy [30,31].

The neurologic outcome of a child with DWM is very variable and depends on the presence of other anomalies of the CNS and the level of control of the hydrocephalus. These patients can present with normal neurological development or be severely compromised. 41 to $71 \%$ of patients can present intelligence coefficients below normal $(\mathrm{IQ}<83)$. However, some studies demonstrated that intelligence can be normal in as many as $75 \%$ of the cases. The severity of the cerebellar dysgenesia also accounts for a worse prognosis, with abnormal vermian lobulation being associated with poor intellectual outcome $[2,30,31,32,33]$.

About 80 to $90 \%$ of children with DWM are diagnosed during the first year of life. Signs and symptoms are related to the hydrocephalus, the cerebellar impairment and cranial nerve dysfunction. It is estimated that $80 \%$ of the patients present ventricles of normal dimensions at birth and $80 \%$ of them will develop hydrocephalus during the first year of life [2,30,31,32,33].

Signs and symptoms of hydrocephalus and increased intracranial pressure are common in children under one year of age. Hydrocephalus is present in $90 \%$ of the patients at the moment of diagnosis and macrocrania is the commonest sign. The increased dimensions of the posterior fossa can lead to bulging of the occiput, with widening of the lambdoid suture that will determine a dolichocephalic appearance of the head. Calvarium defects and occipital meningoceles have also been reported in patients with DWM and the existence of theses abnormalities may be related to the increased pressure in the posterior fossa during fetal life (Fig. 3). As well, some authors report the association between DWM and syringomyelia that probably occur due to the herniation of the cyst through the foramen magno changing the CSF circulation, similar to what happen in the Chiari I malformation [2,36,37].

Children over one year of age usually present with cognitive and developmental delay with gait, coordination and equilibrium being affected. Neurologic deficits such as spastic paraparesia, hypotonia, truncal ataxia, poor fine motor control, dysmetria, speech difficulties, nystagmus, visual and auditory disorders. Seldom is there interference with brainstem respiratory centers, leading to respiratory abnormalities [2].

Seizures occur in 15 to $30 \%$ of patients and are frequently associated with the presence of other CNS malformations, such as heterotopias [34,35].

Morbidity of DWM may be associated with the presence of other systemic malformations, many times related to genetic syndromes. In a European study of 572 cases of DWM, $11 \%$ of the patients presented cardiac defects, 5.9\% anomalies of the urinary tract and $4.6 \%$ limb malformations. Most common structural anomalies were ventricular septal defects, cleft lip with or without cleft palate, atrial septal defect, hypospadias and polydactyly $[30,38]$.

The association of DWM and other brain malformations occurs in 45 to $68 \%$ of autopsy and clinical cases. Anomalies of the corpus callosum are the most frequent ones, affecting up to $10,3 \%$ of the cases. They are followed by aqueduct stenosis, rachischisis, microcephaly, holoprosencephaly, ventriculomegaly and occipital encephalocele [30,38].

Most common chromosomal anomalies that may be associated with DWM are: trisomy 18 (Edwards syndrome), trisomy 13 (Patau's) and $3 q$ syndrome. Among the genetic syndromes, there is an association with Meckel-Gruber, FACE, Klippel-Feil and Mohr syndromes [20].

Imaging studies

The diagnosis of DWM can be made from the 14th week gestational age with the help of the ultrasonography. The mean gestational age at the time of the prenatal diagnosis is 20 weeks, with a large variability among different studies (10 to 38 weeks gestational age). Characteristic findings of DWM in the prenatal ultrasonography are the presence of a large cyst of the posterior fossa associated with the absence of cerebellar vermis and splayed cerebellar hemispheres. These findings help the differential diagnosis with other cystic malformations of the posterior fossa $[39,40]$.

Once the diagnosis of DWM is suspected in the ultrasonography, a magnetic resonance imaging (MRI) should be obtained (Fig.4). Fetal MRI increases the accuracy of the diagnosis, allows for the visualization of the position of the torcula and may demonstrate other associated malformations [20,41].

However several authors claim that fetal MRI seldom adds significant new information, if compared to an ultrasonography of good quality. And even if fetal MR images of excellent quality can be obtained, the anatomical analysis of the vermis is compromised, as neither the primary fissure, nor the pre-pyramidal fissure or its lobulation can be identified with confidence $[42,43]$.

After birth, MRI is the gold standard for the study of posterior fossa lesions. Nevertheless, plain skull $x-$ rays may demonstrate the enlargement of the posterior fossa and elevation of the groove for the transverse sinus and torcula, characteristic features of the DWM. MRI has advantages over computed tomography (CT) scan: there is no exposition to ionizing radiation for children, multiplanar imaging acquisition and better spatial resolution (Fig.5) $[2,20,43]$.

The main imaging findings of DWM are enlarged posterior fossa associated with cystic dilation of the fourth ventricle and superiorly rotated hypoplastic caudal cerebellar vermis. The cystic component of the fourth ventricle occupies the majority of the posterior fossa, compressing the cerebellar hemispheres against the petrous bone. The wall of the cyst may be 
difficult to identity, even in MRI. However, the use of the FIESTA sequence may help identify some of the wall. A compressed and anteriorly displaced 4th ventricle lacking a communication with a cystic extraaxial area is usually the hallmark for the distinction between DWM and arachnoid cysts in the posterior fossa [20].

MRI allows the detection of associated brain malformations, the degree of cerebellar dysgenesis and the analysis of the patency of the aqueduct of Sylvius. Some of the brain malformations include callosal dysgenesis, heterotopia, polymicrogyria, schizencephaly, encephalocele, intracranial lipoma and other conditions [20].

The advances in MRI technology have led to a better understanding of CSF flow disorders. Flowsensitive MRI techniques and three dimensional highresolution sequences have been applied for functional and anatomical assessment of CSF flow dynamics, respectively. These studies can discriminate between communicating and non-communicating hydrocephalus with an accurate localization of the obstruction. For DWM, the determination of CSF flow changes is essential, not only for the differential diagnosis, but also for the indication of the treatment for the hydrocephalus. Phase-contrast sequences have value in studying CSF flow between CSF spaces in the posterior fossa and the posterior cervical subarachnoid space. In the classic DWM, the phasecontrast study demonstrates no flow between the posterior fossa cystic space and the posterior fossa cervical subarachnoid space. Conversely, in the cases of Dandy-Walker variant there is CSF flow between the large retrocerebellar cisterna and the posterior subarachnoid space $[20,44,45]$.

Blake's pouch cyst appears as an infracerebellar or retrocerebellar arachnoid cyst associated with tetraventricular hydrocephalus. Cine-phase MR imaging shows CSF flow through the aqueduct but no clear communication between the fourth ventricle and the posterior fossa cyst, or between the cisterna magna and the posterior cervical subarachnoid space $[19,41,44]$.

A posterior fossa arachnoid cyst appears as a welldefined extra-axial cyst or fluid collection that is associated with a normal vermis. MR imaging shows no CSF flow between the cysts and the posterior cervical subarachnoid space $[2,20,44]$.

A mega cisterna magna corresponds to a focal enlargement of the posterior and inferior subarachnoid space of the posterior fossa, associated with a normal cerebellar vermis and a fourth ventricle of normal size. Phase-contrast MRI reveals free communication between the fourth ventricle and the posterior cervical subarachnoid space $[2,20,44]$.

\section{Pathophysiology of Hydrocephalus}

Hydrocephalus occurs in over $80 \%$ of patients with DWM and the majority presents signs/ symptoms in the first three months of life. Despite the high incidence in patients with DWM, hydrocephalus is not one of the diagnostic criteria for the disease $[2,31,32,33]$.

In the initial description of Dandy and Blackfan, the atresia of the foramina of Luscka and Magendie easily explained the development of hydrocephalus. However, soon after it was observed that the foramina occasionally were patent and that hydrocephalus was not always present at birth. Conversely, atresia of one or two foramen is common in the normal brain, balanced out by the patent foramen. In addition, in DWM the exit of the fourth ventricle may be patent, partially or completely blocked $[3,6]$.

Hydrocephalus in DWM can be considered as multifactorial. In order to explain the complexity of the pathogenesis, Gibson termed this type of hydrocephalus as partially communicating [8].

The aqueduct stenosis, though absent in the classic definition of Barkowich's type A, can be one of the causes of hydrocephalus in patients presenting DWM. The aqueduct stenosis can be either a consequence of the developmental malformation itself, or the result of the herniation of the vermis or the cyst through the tentorial notch. Raimondi called attention to latter and termed it functional aqueduct stenosis: the upward herniation of the posterior fossa contents due to the difference in pressure between the supratentorial compartment and the posterior fossa cyst. This situation ensues when there is overdrainage of the supratentorial ventricular system or cyst shunt malfunction $[2,14,46]$.

Nevertheless several studies have demonstrated the patency of the aqueduct. Asai et al. in 1989 noted patency of the aqueduct in 15 patients who were submitted to a pre-operative ventriculography or MRI. In another retrospective study, the same authors concluded that only 2 of 35 patients with DWM presented with aqueduct stenosis. In a series of patients reported by Mohanty et al. in 2006, the aqueduct was patent in 18 of 21 patients submitted to a pre-operative MRI. According to Spennato at al., these studies allow the conclusion that in the genuine DWM, the aqueduct should be considered patent still proven otherwise [2,47,48,49,52].

Another explanation for the hydrocephalus is that a decrease in CSF flow through the foramina of the fourth ventricle could lead to arachnoiditis in the cisterna magna, perimedullary and incisural cisterns that would block CSF flow. These findings could be attributed to an inflammatory process or the abnormal development of the subarachnoid space as part of the malformation itself [2].

Spennato et al. still consider venous hypertension as an important factor in the genesis of the hydrocephalus. The elevation of the tentorium, torcula and transverse sinus may lead to an elongation of these venous sinuses. Together with direct compression by the posterior fossa cyst with no cerebellar parenchyma to act as a buffer, they would 
lead to venous hypertension and resulting hydrocephalus [2].

Hydrocephalus treatment in Dandy-Walker
malformation

The goal of the treatment of DWM is control of the hydrocephalus and of the posterior fossa cyst. Much debate exists in the literature about the best treatment for DWM. In the last three decades, surgical management has shifted from craniectomy and membrane excision to shunt placement and neuroendoscopic procedures $[2,31,32,47,48,49,50]$.

Dandy and Blackfan in 1914 and Taggart in 1942 proposed direct cyst excision through a craniectomy. However, the outcomes were not good, mortality rates were high and this procedure was rapidly abandoned. The method's failure could be attributed not only to surgical conditions at the time, but also to possible regrowth of the cyst membrane and the limited CSF absorptive capacity of the subarachnoid space in children $[2,48,49,50]$. Today, membrane excision is recommended for the more difficult cases, as those with multiple shunts and frequent shunt malfunctions or cases complicated by infections. For those cases of shunt failure, membrane excision can reduce or optimize the use of multiple shunts or even render the patient shunt independent. This procedure can also be considered a therapeutic alternative to shunt placement in older children. It should be emphasized that, for those cases with the diagnosis of aqueduct stenosis, cyst membrane excision will not be sufficient and aqueductoplasty with the placement of a stent communicating the cyst to the third ventricle will be necessary (Fig.6)[2,48,49,50].

Shunt placement is the surgical procedure of choice in the treatment of hydrocephalus in cases of DWM. However, there is much discussion about the best type of shunt: VP shunt, cyst-peritoneal (CP) shunt or a combination of both? $[2,48,49,50]$.

In the early $90 \mathrm{~s}$, some authors already recommended that surgical approach should be planned according to the extent of obstruction of the aqueduct and subarachnoid space $[2,48,49,50]$.

VP shunts are easy to insert, have a low incidence of catheter malposition or migration and are recommended for those cases presenting with significant hydrocephalus. Early treatment of hydrocephalus is essential for a satisfactory cognitive development and a VP shunt is sufficient for the majority of cases $[2,48,49,50]$. Bindal at al did not find statistically significant differences between the outcome of children with CP shunts and those with VP shunts. They still recommend VP shunts as the treatment of choice, since the complication rates are smaller than those of CP shunts [34,35]. Gerstezen, Albright and Mohanty et al. also share the same opinion. Notwithstanding, decompression of the supratentorial ventricles may lead to upward herniation and acquired aqueduct stenosis, resulting in an isolated fourth ventricle. Cases with isolated fourth ventricle need additional CP shunt placement $[48,49]$.

Other authors favour the choice of CP shunts as the treatment of choice. A CP shunt drains both the cyst and the ventricles adequately in the majority of cases. Yet, CP shunts are associated with a series of complications: difficult placement of the proximal tip, frequent disconnection and migration, posterior fossa hematomas, brainstem tethering, the need for frequent shunt revisions and an increase of CSF fistulas in the postoperative period $[2,48,49]$.

Still other authors advocate the use of a combination of VP and CP shunts to equalize the pressure through the tentorium. Osenbach and Menezes report a success rate of $92 \%$ using a combination of shunts. Simultaneous drainage of the lateral ventricles and the posterior fossa cyst can also be obtained utilizing separate proximal catheters connected by a " $Y$ " connector to a single distal valve and peritoneal catheter, procedure supported by other authors. It is important to note that combined shunt placement can cause the development of secondary aqueduct stenosis, as CSF flow through the aqueduct is greatly reduced. These authors suggest that the diagnosis of aqueduct stenosis demonstrated in the imaging exams, should be the determining factor in the choice of treatment. Cases of aqueduct stenosis require simultaneous drainage of supra and infratentorial compartments to prevent a pressure gradient $[2,47,48,49,50]$.

As endoscopic techniques advance, endoscopic third ventriculostomy (ETV) is a new option for treating DWM. Mohanty et al reported their results of a series of 21 DWM patients treated with ETV. During followup period, only 5 of 21 required an additional VP shunt to treat the hydrocephalus. ETV was the procedure of choice in cases with a patent aqueduct. In cases of aqueduct obstruction, placement of a ventricle-cyst stent was combined with the ETV. This combined treatment was performed to ensure a clear communication between the supratentorial and infratentorial compartments, thus preventing the development of a transtentorial herniation $[48,49]$. In cases of obstructive hydrocephalus, the success rate of ETV ranges from 23 to $80 \%$. The lower rates are seen in infants and young children. After a successful ventriculostomy a slight reduction (16-35\%) in ventricular size has been documented in several studies. The substantial residual ventriculomegaly seen after a successful ETV may prevent secondary aqueduct stenosis, that has been reported to occur due to marked reduction in ventricular size following VP shunt insertion. In this study by Mohanty, secondary aqueduct stenosis was not identified during the follow up period. In 1999, Mohanty et al. already reported the successful use of ETV for cases of DWM with obstruction of the fourth ventricle. No case of isolated fourth ventricle was observed [48,49]. 
In 2011, Warf at al. conducted a retrospective review of 45 patients with DWM who underwent ETV and choroid plexus cauterization and found a total success rate of $74 \%$. Theses authors strongly recommended endoscopic surgery as a primary management method for treating DWM [51].

Kawaguchi et al., in 2003, described the direct observation of the aqueduct and the posterior fossa cyst utilizing a flexible neuroendoscope as an tool for planning treatment. They reported the case of a 3month-old baby girl with DWM treated only with the insertion of a VP shunt after patency of the aqueduct was confirmed [52].

Therefore, all studies agree that the choice of treatment depends on the association of hydrocephalus and the diagnosis of aqueduct stenosis. The presence of aqueduct obstruction warrants simultaneous drainage of the supra and infratentorial compartments to prevent a transtentorial pressure gradient. In the cases a shunt is inserted, the placement of the proximal end of the shunt either into the cyst, or the ventricular cavity, or simultaneous drainage of both cavities is determined by the association of aqueduct obstruction $[2,48,49]$.

With popularity of endoscopic treatment rising, ETV can be the initial treatment of choice for patients with DWM and a patent aqueduct. For the less frequent cases of an association of DWM and aqueduct stenosis, the communication between the two compartments will require an aqueductoplasty or the insertion of a stent in addition to the ETV (Fig.7) In this way, a VP or a CP shunt may be considered if ETV fails $[48,49]$.

These surgical strategies are summarized in table1.

Table 1. Current surgical strategies for Hydrocephalus in Dandy Walker Malformation.

\begin{tabular}{llll} 
Aquedutal Stenosis & $\begin{array}{l}\text { IV Ventricle } \\
\text { Subarachnoid } \\
\text { space }\end{array}$ & Shunts & $\begin{array}{l}\text { Neuroendoscopic } \\
\text { Techniques }\end{array}$ \\
\hline Yes & Unobstructed & VPS & ETV \\
Yes & Obstructed & VPS+CPS & ETV+Aqueducplasty \\
No & Unobstructed & VPS or CPS & - \\
No & Obstructed & VPS or CPS & ETV \\
\hline
\end{tabular}

VPS = ventriculoperitoneal shunt; CPS = cystoperitoneal shunt; $\mathrm{ETV}=$ endoscopic third-ventriculostomy

In conclusion, the frequent episodes of shunt malfunction, complications and dependency, reinforce the idea of ETV as the treatment of choice for hydrocephalus in patients with DWM, especially for older patients or those presenting shunt complications. Still, it is known that patients under 6 months of age have higher ETV failure rates; ventricles and cyst can remain dilated and impair intellectual development at this age, which will favour the insertion of a shunt as the initial treatment in this age range $[48,49]$.

\section{Outcome}

Patients with DWM may develop severe neurological deficits or even death if the hydrocephalus is not treated early and adequately. The commonest causes of death are infection, nontreated hydrocephalus and shunt complications. 50\% of children with DWM and hydrocephalus that is not treated die before the age of 3 years of age. Only 20$23 \%$ reaches adulthood and most present visual, auditory or motor deficits. Seizures occur in about $30 \%$ of the patients with hydrocephalus and is a marker of worse outcome [53,54].

Sixteen percent of patients with isolated DWM have chromosomal alterations and are associated with malformations of face, limbs, cardiovascular, genitourinary or gastrointestinal systems that, furthermore, affects the outcome and increases morbidity of patients $[30,33]$.

Similarly, intellectual outcome also depends on other associated malformations of the CNS. In 2006, Boddaert and Klein showed that the degree of damage of the vermis was directly related to the intellectual development [55].

\section{Conclusion}

The Dandy-Walker malformation is a rare pathological entity still insufficiently know under etiologic and physiopathological aspects. The correct diagnosis of Dandy-Walker malformation can be made through careful interpretation of magnetic resonance imaging (MRI) and cerebrospinal fluid (CSF) flow studies. Hydrocephalus occurs in over $80 \%$ of patients with DWM. The choice of hydrocephalus treatment depends on whether there is aqueduct stenosis. And, although ventriculoperitoneal (VP) shunts have been the treatment of choice for many years, neuroendoscopic techniques such as endoscopic third ventriculostomy and stent placement are being frequently performed lately.

\section{Disclosure Statement}

The authors have no conflicts of interest to declare.

\section{Funding statement}

None.

\section{References}

1. Sutton JB. The lateral recesses of the fourth ventricle: Their relation to certain cysts and tumors of the cerebellum and to occipital meningocele. Brain. 1887; 9: 352-361. 
2. Spennato P, Mirone G, Nastro A et al. Hydrocephalus in Dandy- Walker malformation. Childs Nerv Syst. 2011; 27: 1665-1681.

3. Dandy WE, Blackfan KD. Internal hydrocephalus: an experimental, clinical, and pathological study. Am J Dis Child. 1914; 8: 406-482.

4. Taggart J, Walker AE. Congenital atresia of the foramens of Luschka and Magendie. Arch Neurol Psychiatry. 1942; 48: 583-612.

5. Sahs AL. Congenital anomaly of the cerebellar vermis. Arch Pathol 1941; 32: 52-63.

6. Benda CE. The Dandy-Walker syndrome so called atresia of foramen Magendie. J Neuropathol Exp Neurol 1954;13: 14-29.

7. Gardner WJ. Hydrodynamic factors in Dandy-Walker and Arnold-Chiari malformations. Child's Brain 3:200212

8. Gibson JB. Congenital hydrocephalus due atresia of the foramen of Magendie. J Neuropathol Exp Neurol. 1955; 14: 244-262.

9. D'Agostino AN, Kernohan JW, Brown JR. DandyWalker syndrome. J Neuropathol Exp Neurol. 1963; 22: 450-470.

10. Hart MN, Malamud N, Ellis WG. The Dandy-Walker syndrome: A clinicopathological study based on 28 cases. Neurology.1972; 22: 771-780.

11. Raybaud C. Cystic malformations of the posterior fossa. Abnormalities associated with the development of the roof of the fourth ventricle and adjacent meningeal structures. J Neuroradiol. 1982; 9:103-133.

12. Gonsette R, Potvliege R, Andre- Balisaux G, Stenuit J. Mega-cisterna magna: Clinical, radiologic and anatomopathologic study. Acta Neurol Psychiatry Belg. 1968; 68: 559-70.

13. Harwood -Nash DC, Fitz CR. Neurorradiology in infants and children. 1976; vol.3.Mosby, St Louis,10149.

14. Barkovich AJ, Kjos BO, Norman D, Edwards MS. Revised classification of posterior fossa cysts and cystlike malformations based on the results of multiplanar MR imaging. AJNR. 1989; 10:977-988.

15. Tortori-Donati P, Fondelli MP, Rossi A, Carini S. Cystic malformations of the posterior cranial fossa originating from a defect of the posterior membranous area. Mega cisterna magna and persisting Blake's pouch: two separate entities. Child's Nerv Syst. 1996; 12:303-308.

16. Kollias SS, Ball WS Jr. Prenger EC. Cystic malformations of the posterior fossa: Differential diagnosis clarified through embryologic analysis. Radiographics .1993; 13:1211-31.

17. Gilles FH, Rockett FX. Infantile hydrocephalus: retrocerebellar aracnhnoid cyst. J Pediatr. 1971; 79: 43643.

18. Blake JA. The roof and lateral recesses of the fourth ventricle, considered morphologically and embryologically. J Comp Neurol. 1900; 10: 79-108.

19. 19.Azab WA, Shohoud SA, Elmansoury TM, et al . Blake's pouch cyst. Surg Neurol Int. 24-Jul-2014; 5:112.

20. Correa GC, Amaral LF, Vendolin LM. Neuroimaging of Dandy- Walker Malformation- New Concept, Topic in Magnetic Resonance Imaging. 2011; 22(6): 303-312.

21. Shekdar K. Posterior fossa malformations. Semin Ultrasound CT MR. 2011; Jun 32 (3):228-41.

22. Johal J, Paulk PB, Oakes PC et al. A comprehensive review of the foramina of Luschka: history, anatomy, embryology, and surgery. Child Nerv Syst published online: 04 july 2017.
23. Tortori-Donati P, Rossi A: Brain malformations, in Pediatric Neuroradiology (vol 1). New York, Springer, 2005.

24. Norman MG, Kalousek DK, Hill A, et al. Congenital Malformations of the Brain: Pathologic, Embryologic, Clinical, Radiologic and Genetic Aspects. New York 1995, Oxford University Press.

25. Yachnis AT. Rhombencephalosynapsis with massive hydrocephalus: Case report and pathogenetic considerations. Acta Neuropathol. 2002; 103: 301-304.

26. Altman NR, Naidich TP, Braffman BH. Posterior fossa malformations. Am J Neuroradiol. 1992; 13:691-724.

27. Friede RL: Developmental Neuropathology (ed 2). Berlin, Springer, 1989

28. Costa C: Pediatric Neuropathology. Baltimore, Williams \& Wilkins, 1995

29. Patel S, Barkovich AJ. Analysis and classification of cerebellar malformations. Am J Neuroradiol .2002; 23:1074-1087.

30. Santoro M, Coi A, Barišić I, et al. Epidemiology of Dandy- Walker Malformation in europeu: A EUROCAT Population-Based Registry Study, Neuroepidemiology. Published online; (1-11) July 12, 2019.

31. Hirsch JF, Pierre-Kahn A, Renier D, et al. The DandyWalker malformation: A review of 40 cases. J Neurosurgery .1984; 61:515-522.

32. Klein O, Pierre-Kahn A. La Malformation de DandyWalker. Neurochirurgie. 2006; 52 (4): 347-356.

33. Zamora EA, Ahmad A. Dandy Walker Malformation in StatPearls [Internet]. Treasure Island (FL): 11 Mar 2019.

34. Bindal AK, Storrs BB, McLone DG. Occipital meningoceles in patients with Dandy- Walker syndrome. Neurosurgery. 1991; 28: 844-847.

35. Bindal AK, Storrs BB, Mc Lone DG. Management of Dandy- Walker Syndrome. Pediatr Neurosurg 1991; 16:163-169.

36. Baro V, Manara R, Denaro L, D' Avella D. Dandy Walker malformation and syringomyelia: a rare association; Child Nerv Syst published online: 18 March 2018.

37. Zhang N, Qi Z, Zhang X, et al. Dandy- Walker syndrome associated with syringomyelia in an adult: a case report and literature review. Journal of International Medical Research 2019; 47(4):1771-1777.

38. Stambolliu E, Iokeim- Ioannidou M, Kontokostas k, Dakoutrou M, Kousoulis AA. The most common comorbidities in Dandy- Walker Syndrome Patients: A systematic review of case reports. J. Child Neurol. 2017; 32 (10): 886-902.

39. Guibaud L, Larroque A, Ville D, et al: Prenatal diagnosis of 'isolated' Dandy- Walker malformation: Imaging findings in prenatal counselling. Prenatal diagnosis 2012; 32: 185-193.

40. Paladini D, Quarantelli M, Pastore G, et al. Abnormal or delayed developement of the posterior membranous área of the brain: anatomy, ultrasound diagnosis, natural history and outcome of Blak's pouch cyst in fetus. Ultrasound Obstet Gynecol. 2012; 39: 279-287.

41. Kau T, Marterer R, Kottke R, et al: Blake's Pouch Cysts and Differential Diagnosies in Prenatal and Postnatal MRI. Clinical Neuroradiol. 15 january 2020, online.

42. Gandolfi CG, Contro E, Carletti A, et al. Prental diagnosis and outcome of fetal posterior fossa fluid collections. Ultrasound Obstet Gynecol. 2012; 39:625631.

43. Malinger G, Lev D, Lerman-Sagie T. Is fetal magnetic resonance imaging superior to neurosonograhy for 
detection brain anomalies? Ultrasound Obstet Gynecol. 2002; 20: 317-321.

44. Mohammad SA; Osman NM; Ahmed KA; The value of CSF flow studies in the management of CSF disorders in children: a pictorial review. Insights into Imaging 2019; 10(3); 1-13.

45. Jurca MA, Kozma K, Petchesi CD, et al. Anatomic variants in Dandy-Walker complex. Rom J Morphol Embryol 2017; 58(3): 1051-1055.

46. Raimondi AJ, Samuelson G, Yazargaray L, Norton T. Atresia of the foramen of Lusckha and Magendie: the Dandy-Walker Cyst. J Neurosurg 1969; 31:202-216.

47. Asai A, Hoffman HJ, Hendrick EB, et al Dandy- Walker Syndrome Experience at the Hospital for Sick Children, Toronto. Pediatr Neurosci. 1989; 15: 66-73.

48. Mohanty A. Endoscopic Third Ventriculostomy with cystoventricular stent placement in the management of Dandy-Walker Malformation: Technical case report of three patients. Neurosurgery. 2003; 53: 1223-1229.

49. Mohanty A, Biswas A, Satish S, Praharaj SS, Sastry KV. Treatment options for Dandy-Walker malformation. J Neurosurg. 2006 Nov;105(5 Suppl):348-56.

50. Lin J, Liang G, Liang Y and Kou Y. Long- term followup of successful treatment for Dandy- Walker syndrome (DWS). Int J Clin Exp Med 2015; 8(10): 18203-18207.
51. Warf BC, Dewan M, Mugamba J. management of Dandy- Walker complex- associated infant hydrocephalus by combined endoscopy third ventriculostomy and choroid plexus cauterization. J Neurosurgery Pediatr. 2011; 8:377-83.

52. Kawaguchi T, Jokura H, Kusaka Y, Shirane R and Yoshimoto $\mathrm{T}$. Intraoperative direct neuroendoscopic observation of the aqueduct in Dandy- Walker malformation. Acta Neurochir. 2003; 145: 63-67.

53. Chumas P, Tyagi A, Livingston J. Hydrocephalus what's new? Arch Dis. Child. Fetal Neonatal. 2001; 85 (3) $149-154$.

54. Donders J, Canady AI, Rourkwe BP. Psychometric intelligence after infantile hydrocephalus. A critical review and reinterpretation. Childs Nerv Syst. 1990; 6 (3): 148-154.

55. Boddaert N, Klein O, Ferguson N, Sonigo P, Parisot D, Hertz- Pannier L, Baraton J, Emond S, Simon I, Chigot V, Schmit P, Pierre-Kahn A, Brunelle F. Intellectual prognosis of the Dandy-Walker malformation in children: the importance of vermian lobulation. Neuroradiology. 2003; 45:320-324. 